\title{
A Controlled Clinical Trial of Continuous and Intermittent Rifampicin Therapy During an Initial Three Months Period in Lepromatous leprosy: Final Analysis
}

\author{
S. R. PATTYN \\ University of Antwerp, UIA, Institute for Tropical Medicine, \\ Antwerp, Belgium \\ M. T. ROLLIER AND R. ROLLIER \\ Service National de léprologie, Casablanca, Maroc \\ E. J. SAERENS AND P. DOCKX \\ University of Antwerp, UIA, Antwerp, Belgium
}

\begin{abstract}
A controlled clinical trial was organized as a 3 months introductory treatment of lepromatous leprosy, comparing the following treatment regimens administered in the hospital: dapsone $100 \mathrm{mg}$ daily, rifampicin $450 \mathrm{mg}$ daily, rifampicin $900 \mathrm{mg}$ once a week and clofazimine $300 \mathrm{mg}$ once a week. Thereafter the patients were discharged, and treated with standard dapsone $100 \mathrm{mg}$ in routine selfadministration. Clinical, biological and microbiological assessments were performed at the start and after 1, 2, 3, 6, 9 months of treatment. BI and MI were determined on a blind basis. One hundred and twenty-nine patients were admitted to the trial, 93 remaining for final analysis. Results show that from a microbiological standpoint the 2 rifampicin groups behaved similarly, their MI reaching minimum levels within one month, as compared with 3 to 6 months in the case of dapsone and clofazimine treated groups, the clofazimine group being the slowest. Clinical improvement was somewhat more rapid in the RMP groups, especially the cicatrization of sof palate ulcerations and in the daily rifampicin group the regression of peripheral anaesthesia. There was somewhat more ENL, although not statistically significant, after rifampicin weekly. There were no other complications associated with any of the treatment schedules. The results show that intermittent once weekly rifampicin treatment is as efficient as daily therapy, and that the period of treatment may even be shortened to 2 months, thus reducing further the total amount of drug administered. Clofazimine $300 \mathrm{mg}$ once a week induces too slow an improvement, although it could be useful as an addition for combined introductory intermittent therapy.

Nasal smears may be as sensitive indicators of the bacteriological evolution under $\mathrm{d}$ rug treatment as are skin biopsies.
\end{abstract}




\section{Introduction}

Results of treatment of chronic infectious diseases can be much improved if treatment can be supervised. Supervision of treatment is practicable only when it is intermittent. Intermittent treatment has a further advantage in reducing the total amount of drug administered.

Acedapsone is at this moment the drug which lends itself to the longest intermittency in the therapy of leprosy. However, as discussed previously (Pattyn, 1972) the administration of acedapsone in the treatment of multi-bacillary forms of the disease should be preceded by an introductory phase that is rapidly bactericidal.

Rifampicin is a rapidly bactericidal drug for Myco. leprae (Rees et al., 1970, Shepard et al., 1972) when administered in daily doses. From experiments in mice it is known (Pattyn e't al., 1974) that it is also active when given intermittently.

We therefore initiated a controlled clinical trial to compare the effect of daily administration of rifampicin with a once weekly administration. A weekly administration of clofazimine was also included in the trial whereas classical dapsone therapy was the reference treatment. Preliminary results on this trial have been published elsewhere (Pattyn et al., 1974).

\section{Organization of the Trial}

\section{Selection of patients}

Patients were selected at the out-patient department of dermatology at Casablanca, where 200 new leprosy patients a year-are seen, nearly half of them suffering from lepromatous or borderline lepromatous forms of disease. These were hospitalized for investigation and eventual inclusion in the trial.

\section{Pretreatment investigations}

The severity of skin involvement was estimated and graded: for erythematous lesions, 2 for infiltrated plaques and 3 for nodular lesions. The degree of anaesthesia of the extremities was also graded into categories: 0 for absence of anaesthesia, 1 for finger or toe anaesthesia, 2 for forearm or leg anaesthesia and 3 for anaesthesia of the whole member.

Routine haematological examinations were performed including red blood cell count, haemoglobin and haematocrit determination, erythrocyte sedimentation rate (ESR), and a chest X-ray was taken. Smears were prepared from the nasal mucosa, the ear-lobes and the skin of the forehead. A skin biopsy from a clearcut lesion was taken and fixed in $10 \%$ formalin.

\section{Criteria for admission}

For inclusion in the trial patients had to conform to the following conditions: to be adults with previously untreated lepromatous or near lepromatous leprosy, not to suffer from concomitant tuberculosis, and to show in their biopsies a Morphological Index (MI) higher than 0.10 to ascertain clearer results. The trial started in May, 1972, the last patients entered the trial in May, 1974. 


\section{Chemotherapeutic regimens}

Using a table of random numbers, patients were admitted to one of the following treatment regimens administered during their 3 months hospitalization.

RMP 450: rifampicin 8-10 mg/kg body.weight $450 \mathrm{mg}$ daily.

RMP 900: rifampicin $15 \mathrm{mg} / \mathrm{kg}$ body weight, $900 \mathrm{mg}$, once a week.

CLO 300: clofazimine $300 \mathrm{mg}$ once a week.

DDS 100: dapsone $100 \mathrm{mg}$ daily (starting with a lower dose and reaching the $100 \mathrm{mg}$ dosage after 2 weeks).

All drugs were administered in the early morning about 1 hour after breakfast.

After 3 months patients were discharged from the hospital and treated by the classical dapsone therapy, $100 \mathrm{mg}$ daily in self-administration. The drug is given to them at each out-patient control visit.

\section{Management of patients}

During the hospitalization period, dermatological, neurological, haematological examinations and skin biopsies were repeated after 1, 2 and 3 months. All biopsies were sent to Antwerp. During the out-patient period, patients were invited to return to the hospital after a further 3 months for examinations as above. Thereafter patients are expected to return to the hospital every 6 months. In this trial patients were followed for at least 6 months.

\section{Bacteriological procedures}

Code numbered biopsies were sent to Antwerp where they were examined after haematoxylin-eosin and Ziehl-Neelsen (Fite-Faraco technique) staining. Bacterial and morphologic indices (BI and MI) were recorded without any knowledge of the treatment schedule or clinical status of the patients. Data of these determinations were communicated and stained slides were sent to Casablanca.

\section{Clinical assessments}

Patients were examined monthly, when weight and ESR were recorded, and clinical improvement and sensibility of the extremities were evaluated. The degree of resolution in lepromatous infiltration was recorded as 0 when unchanged, 1 for discrete improvement, 2 for easily visible improvement, 3 for a reduction in infiltration by $50 \%, 4$ for nearly complete resolution, and 5 for a total disappearance of any cutaneous signs, "W" if the condition was worsening or if erythema nodosum (ENL) developed.

The occurrence of ENL was also noted during the follow-up period, as were all other complaints made by the patients.

\section{Results}

\section{Exclusions}

A total of 129 patients participated in the trial (Table 1). From these, 19 had to be excluded from the final analysis because of a MI below 0.10 at the start of 
treatment, 16 patients had to be excluded because a group of biopsies was lost during air transport and finally 1 was excluded because the clinical file was unavailable.

Table 2 shows the distribution of the 93 patients remaining for final analysis within each treatment group. The number of males largely exceeds the number of females, reflecting the difference in sex distribution of leprosy as seen in Morocco. Mean age, body weight and ESR in each group are comparable (Table 3). The degree of skin involvement (Table 4) and anaesthesia was moderate in most patients and not significantly different between the different treatment groups.

\section{TABLE 1}

Population studied

\begin{tabular}{lccc}
\hline Patients admitted into trial & $\begin{array}{c}\text { Male } \\
95\end{array}$ & $\begin{array}{c}\text { Female } \\
34\end{array}$ & $\begin{array}{c}\text { Total } \\
129\end{array}$ \\
\hline Excluded from analysis: & & & \\
MI 0.10 & 14 & 5 & 19 \\
Biopsies lost by accident & 13 & 3 & 16 \\
Files unavailable & 1 & & 1 \\
Total remaining for analysis & 67 & 26 & 93 \\
\hline
\end{tabular}

TABLE 2

Distribution of patients among treatment groups

\begin{tabular}{lcccc}
\hline & RMP 450 & RMP 900 & CLO 300 & DDS 100 \\
\hline Number of patients & \multicolumn{1}{c}{25} & 25 & 21 & 22 \\
male : female & $15: 10$ & $18: 7$ & $17: 4$ & $17: 5$ \\
Average age (male) & 35.8 & 32.3 & 40.4 & 39.0 \\
$\quad$ max - min. & $65-14$ & $55-16$ & $61-16$ & $72-20$ \\
Average age (female) & 38.7 & 25.7 & 42.2 & 42.4 \\
max - min. & $62-15$ & $53-17$ & $50-15$ & $75-17$ \\
\hline
\end{tabular}

TABLE 3

Weight and ESR of patients

\begin{tabular}{lcccc}
\hline & RMP 450 & RMP 900 & CLO 300 & DDS 100 \\
\hline Weight average & 53.6 & 55.3 & 55.0 & 55.1 \\
& $80 / 37$ & $92 / 39$ & $80 / 39$ & $70 / 41$ \\
ESR average & $62 / 87$ & $42 / 66$ & $54 / 85$ & $60 / 85$ \\
Extremes max. & $140 / 144$ & $115 / 130$ & $121 / 142$ & $130 / 135$ \\
Extremes min. & $7 / 18$ & $5 / 12$ & $4 / 10$ & $5 / 15$ \\
\hline
\end{tabular}


TABLE 4

Degree of skin involvement and anaesthesia of extremities

\begin{tabular}{lrrrrr}
\hline & & RMP 450 & RMP 900 & CLO 300 & DDS 100 \\
\hline Degree of skin involvement at start & 1 & 9 & 3 & 5 & 4 \\
& 2 & 15 & 21 & 15 & 18 \\
Degree of anaesthesia at start & 3 & 1 & 1 & 1 & - \\
& 0 & 7 & 8 & 4 & 6 \\
& 1 & 16 & 17 & 14 & 12 \\
& 2 & 2 & & 3 & 4 \\
\hline
\end{tabular}

\section{Evolution of $M I$ and $B I$}

Figure 1 shows the evolution of BI and MI during 6 months for each regimen. The mean MI before treatment was slightly lower in the DDS and CLO groups. The evolution of MI in the 2 RMP regimens differs significantly from that in the other 2 regimens: it fell down under 0.10 within 1 month for both RMP regimens while this is only the case after 2 months for DDS 100 and 3 months for CLO 300. Minimal values for the MI were reached after 1 month for RMP 450, 2 months for RMP 900, but not within 3 months for the CLO and DDS groups.

In the preliminary data previously published (Pattyn et al., 1974) mention was made of a comparison of the MI determined independently in Antwerp and Casablanca. A small difference was noted between the 2 readings but the general trend was entirely comparable. The complete figures confirm the preliminary findings: values were slightly higher in Casablanca, but the evolution was the same (Fig. 2). The BI in the nose smears (Table 5) also fell more rapidly in the 2 RMP regimens: from more than 4.0 before treatment to 2.0 within 1 month. However,

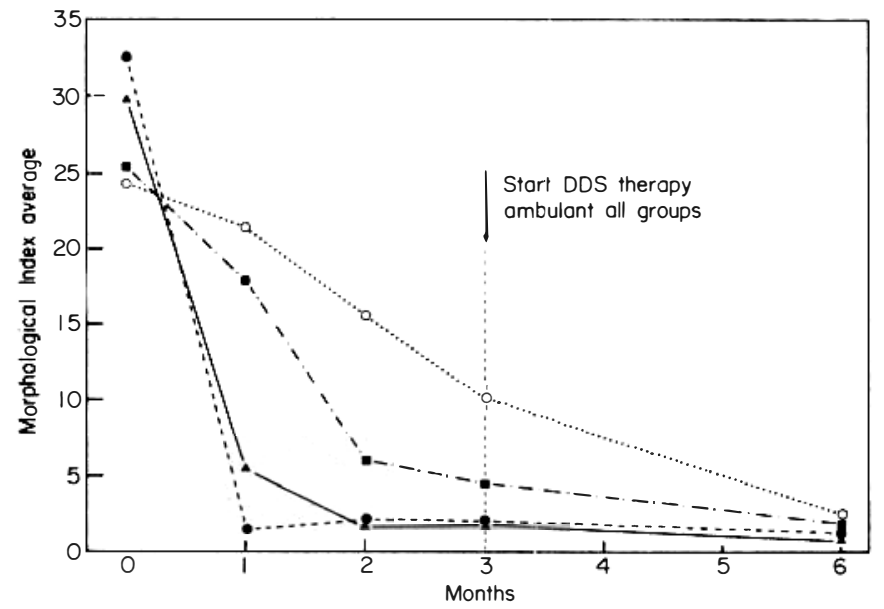

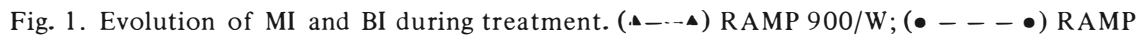
450/D; (- - • DDS 100/D; ( … o) CLO 300/W. 


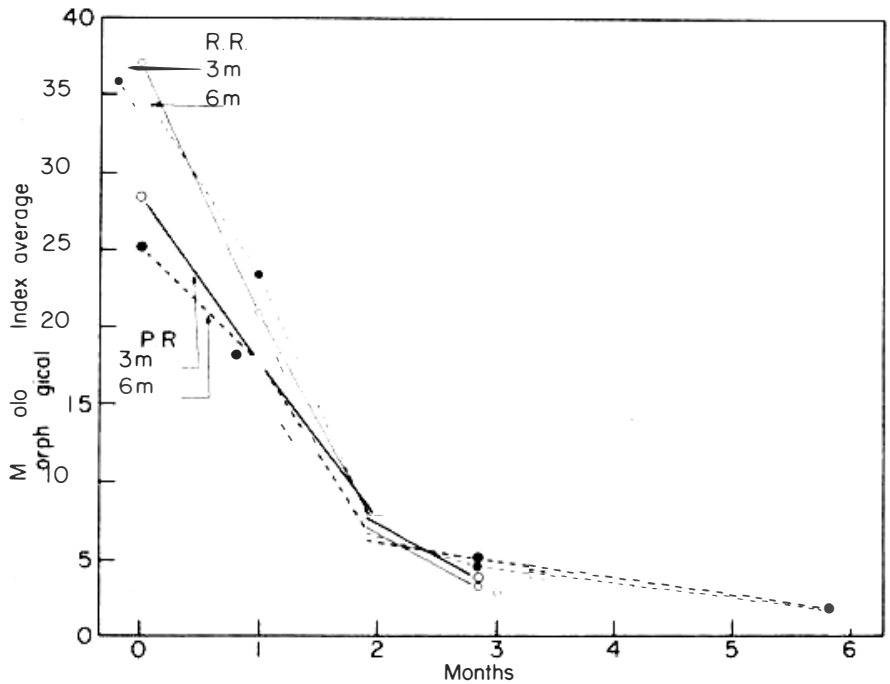

Fig. 2. Comparison of 2 different biopsy evaluations (at 3 and 6 months) R. R. Rollier reading; P. R. Pattyn reading.

TABLE 5

Evolution of BI and MI in nose smears

\begin{tabular}{lcccc}
\hline & RMP 450 & RMP 900 & CLO 300 & DDS 100 \\
\cline { 1 - 2 } \multicolumn{1}{c}{ BI } & & & & \\
Start & 4.7 & 4.4 & 3.8 & 3.2 \\
1 month & 2 & 1.9 & 3.6 & 3.1 \\
2 months & 1.8 & 2.4 & 3.3 & 2.5 \\
3 months & 1.7 & 1.5 & 2.6 & 1.9 \\
$\quad$ MI & & & & \\
Start & 0.34 & 0.34 & 0.22 & 0.20 \\
1 month & 0.01 & 0.02 & 0.18 & 0.20 \\
2 months & $<0.01$ & 0.01 & 0.05 & 0.09 \\
3 months & $<0.01$ & $<0.01$ & 0.02 & 0.01 \\
\hline
\end{tabular}

after 3 months there is no notable difference between the RMP and DDS treated patients. Again the CLO 300 group lags behind in this respect also.

As was the case in the skin the MI in the nose smears at the start was somewhat higher in the RMP treated patients. Within 1 month the MI in these patients reached baseline levels, while this situation was reached in the CLO and DDS groups at the third month only. The BI in the skin did not change during the observation period (Table 6).

\section{Clinical evolution}

Due to circumstances, some clinical data are lacking, reducing the number of patients available for this analysis. Table 7 shows the evolution of body weight 
TABLE 6

Evolution of BI during treatment (mean values)

\begin{tabular}{lcccc}
\hline & RMP 450 & RMP 900 & CLO 300 & DDS 100 \\
\hline Start & 4.7 & 4.5 & 4.8 & 4.5 \\
1 month & 4.7 & 4.3 & 4.5 & 4.2 \\
2 months & 4.1 & 4.7 & 4.3 & 4.3 \\
3 months & 3.6 & 4.5 & 4.7 & 4.3 \\
6 months & 4.1 & 4.1 & 4.1 & 4.3 \\
\hline
\end{tabular}

TABLE 7

Evolution of weight and ESR during treatment

RMP $450 \quad$ RMP $900 \quad$ CLO $300 \quad$ DDS 100

\begin{tabular}{lllll}
\hline Weight average & & & & \\
Start & 53.6 & 55.3 & 55.0 & 55.1 \\
1 month & 53.8 & 55.5 & 55.3 & 55.8 \\
2 months & 55.0 & 56.8 & 54.1 & 56.7 \\
3 months & 55.6 & 57.4 & 54.2 & 57.3 \\
ESR average & & & & \\
Start & $62 / 87$ & $42 / 66$ & $54 / 85$ & $60 / 85$ \\
1 month & $64 / 88$ & $51 / 69$ & $57 / 80$ & $56 / 84$ \\
2 months & $54 / 80$ & $57 / 82$ & $67 / 90$ & $63 / 87$ \\
3 months & $56 / 79$ & $52 / 72$ & $65 / 85$ & $63 / 88$ \\
\hline
\end{tabular}

and ESR. There is some weight increase in all treatment regimens except for CLO 300 while the values for ESR are lower for the RMP 450 only, these differences are not significant.

Figure 3 shows the histogram, depicting the degree of reduction of infiltration of the cutaneous lesions. The fastest rate of improvement is observed in the RMP 450 regimen, followed by the RMP 900 and DDS 100 regimens, while the CLO 300 regimen leads manifestly to a slower improvement. There is a parallelism between the fall of the $\mathrm{MI}$ in the different groups and the rate of cutaneous improvement.

As can be seen from Table 8, improvement of anaesthesia of the extremities was much faster in the RMP 450 regimen: at 6 months 17 out of 19 patients showed a considerable improvement in this respect, while this was only so for roughly half the patients on the other 3 regimens. Table 9 shows the number of ENL episodes during the first 3 months, the next 3 months and later. One patient in each of the RMP 900 and CLO 300 groups was suffering from this condition at the start of treatment. There was a non-significant greater number of ENL episodes during the first 6 months of treatment within the RMP 900 group; the numbers of ENL within the other groups are absolutely comparable.

At the start of treatment a considerable number of patients had rhinitis, even haemorrhagic (Table 10). This condition also improved more rapidly among the RMP treated patients, but this is difficult to quantify and is therefore not 


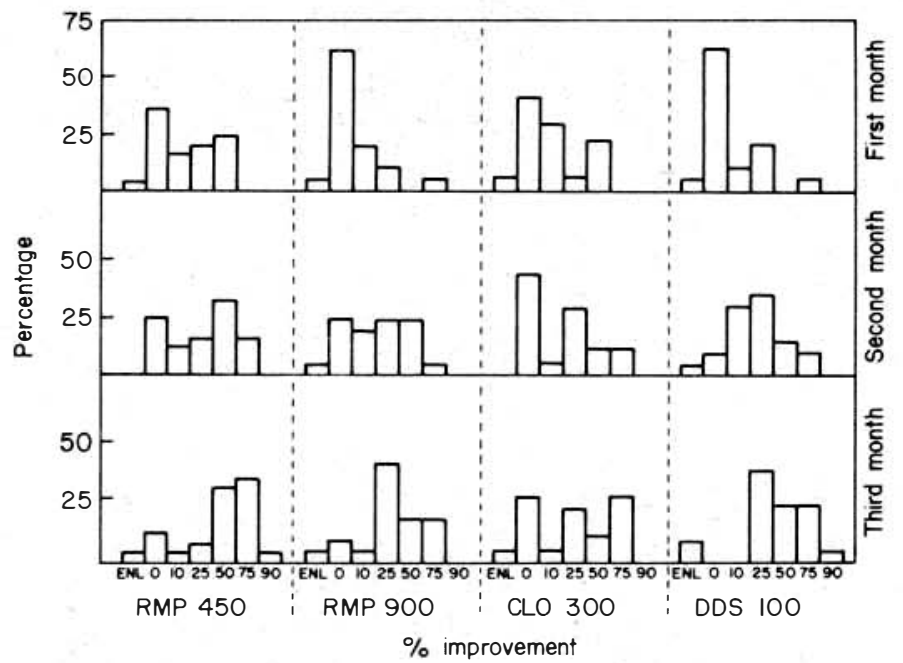

Fig. 3. Clinical improvement during first 3 months of treatment.

TABLE 8

Evolution of anaesthesia of extremities during the first 3 months of treatment

\begin{tabular}{lcccc}
\hline & Improvement & Status quo & No. at start & Total \\
\hline RMP 450 & 16 & 2 & 6 & 24 \\
RMP 900 & 9 & 6 & 8 & 23 \\
CLO 300 & 7 & 7 & 4 & 18 \\
DDS 100 & 9 & 7 & 6 & 22 \\
\hline
\end{tabular}

TABLE 9

Incidence of ENL

\begin{tabular}{lcccc}
\hline & RMP 450 & RMP 900 & CLO 300 & DDS 100 \\
\hline \multicolumn{1}{c}{ ENL } & & & & \\
Start & 1 & 0 & 1 & 0 \\
First 3 months & 2 & 3 & 1 & 2 \\
4-6 months & 1 & 6 & 3 & 3 \\
After 6 months & 1 & 1 & 1 & 3 \\
\hline
\end{tabular}

recorded. Ulcerations of soft palate were present in some patients, cicatrisation of these was clearly more rapid within the RMP 900 group, while in the CLO 300 and DDS 100 groups, these lesions were not healed in some patients after 3 months of treatment.

\section{Other complications}

Table 11 shows all complications and the subjective complaints noted during the first 3 months of treatment. Especially important here, are symptoms which 
TABLE 10

Evolution of nose lesions

\begin{tabular}{lcccc}
\hline & RMP 450 & RMP 900 & CLO 300 & DDS 100 \\
\hline Start & & & & \\
$\quad$ Total & 25 & 25 & 21 & 22 \\
$\quad$ Rhinitis & 14 & 13 & 9 & 10 \\
$\quad$ Haemorrh. rhin. & 11 & 9 & 10 & 12 \\
$\quad$ Palate ulcer. & 7 & 5 & 4 & 8 \\
Palate cicatr. & 4 & 5 & 1 & 6 \\
$\quad$ month & 1 & & & \\
2 months & 1 & & & \\
$\quad$ m months & 1 & & 2 & \\
unknown & & & & \\
\hline
\end{tabular}

TABLE 11

Clinical complications during the first 3 months of treatment

\begin{tabular}{lcccc}
\hline & RMP 450 & R MP 900 & CLO 300 & DDS 100 \\
\hline $\begin{array}{l}\text { Arthralgia } \\
\text { Fever (headache) }\end{array}$ & 3 & 1 & 2 & 1 \\
Jaundice & 1 & 1 & 1 & 1 \\
$\begin{array}{l}\text { Morbilliform erythema } \\
\text { Neuralgia }\end{array}$ & 1 & 2 & & 1 \\
$\begin{array}{l}\text { Pruritus } \\
\text { Stomach pains }\end{array}$ & & & 2 & 1 \\
$\begin{array}{l}\text { Bronchitis } \\
\text { Cystitis } \\
\text { Increased ESR at 2 months }\end{array}$ & & 1 & 1 \\
Zona & & 1 & \\
\hline
\end{tabular}

could be expressions of complications of the intermittent administration of RMP. Serious complications such as thrombocytopenia and anuria were not observed. In no instance had the therapeutic regimen to be interrupted or changed. One case of icterus occurred in the DDS group after 2 months treatment. Arthralgias and febrile episodes occurred as frequently in those patients not receiving as those taking RMP. One patient on dapsone had a sudden unexplained increase in his ESR at 2 months, which disappeared at 3 months.

\section{Discussion}

The evolution of the reference dapsone treatment is as could be expected from studies previously performed by other authors (Shepard et al., 1968; Rees, 1965) 100 days of treatment being necessary to obtain maximal measurable bactericidal effect.

The results of the RMP 450 regimen are also consistent with previous experience, namely a rapid decline of the MI, within 1 month of treatment (Rees et al., 1970; Shepard et al., 1972; Pattyn et al., 1972). Our results show that with 
patients whose average weight does not exceed $60 \mathrm{~kg}$, a dosage of $450 \mathrm{mg}$ daily may be sufficient. Intermittent administration of RMP, $900 \mathrm{mg}$ once weekly produces an identical decline of the MI in skin biopsies and nose smears, and the same fall in the BI in the latter. The regression of anaesthesia was significantly more rapid in the daily RMP regimens, compared with the three other groups.

The greater number of ENL episodes observed with the RMP 900 regimen. especially during the second trimester after the start of treatment, is not statistically significant. This should be investigated on a greater number of patients.

The weekly administration of RMP was not associated with any significant sideeffect. This must be attributed to the relatively low dose of the drug administered and the short duration: most side-effects have been observed after more than 3 mon ths intermittent therapy (Gyselen, 1971).

The total amount of rifampicin administered during 3 months in the RMP 900 regimen has been but one fourth of the total amount administered in the continuous regimen: $10.8 \mathrm{~g}$ compared with $40.5 \mathrm{~g}$. The results of the present trial, and those of experiments in mice (Pattyn and Saerens, 1974) allow the conclusion that the intermittent administration of RMP may be reduced to a period of 2 months, or a total amount of $7.2 \mathrm{~g}$.

The important question of necessity for combined therapy during the initial phase was not considered in this short term trial and can only be resolved by very long term trials (Committee on experimental therapy, 10th International Congress for Leprosy, Bergen 1973). An effort will be made to follow as long as possible the patients who participated in the present trial.

From a theoretical point of view and in analogy with the situation in tuberculosis (Pattyn, 1972) combined drug therapy is probably unavoidable in the initial phase of treatment of multibacillary leprosy. Since there is no antagonism between dapsone and rifampicin in their activity on Myco. leprae (Shepard, 1973), one could probably administer acedapsone from the very start and even add weekly injections of thiambutosine for 2 or 3 months, provided this does not result in clinical complications. This was not done in the present trial because it was first necessary to assure that the weekly RMP regimen compared favourably with the daily administration of the drug.

Clofazimine in a dosage of $300 \mathrm{mg}$ once weekly was clearly the least effective treatment both in terms of bacteriological results as in clinical improvement. But it might be useful as a component of the initial combined introductory treatment referred to above. One other important result of the present study is that nasal smears may constitute a parameter as important as skin biopsies to monitor the effect of drug treatment of multibacillary forms of the disease. Although it is difficult to standardize nasal smears, their BI diminished significantly within the first months of treatment with RMP and reached comparable values after 3 months in all treatment groups. This may be in direct relationship with the healing of the rhinitis in lepromatous leprosy. In the clofazimine treated patients the MI in the nose smears fell significantly more rapidly than in the skin. One may wonder if this is the result of a concentration of the drug in the nasal mucosa.

The treatment of multibacillary leprosy can be conducted in an analogous way as is presently done for tuberculosis: a short term introductory therapy rapidly bactericidal, intermittent (preferably combined) and therefore supervisable, followed by long term intermittent treatment with acedapsone, which should provide fool proof treatment of the disease. 


\section{Acknowledgement}

This work was supported by a grant from the Damiaanfonds, Brussels, Belgium.

\section{References}

Pattyn, S. R. (1972). Comments on the chemotherapy of leprosy as influenced by present knowledge of M. leprae. Lepr. Rev. 43, 126-136.

Pattyn, S. R., Rollier, R., Rollier, M. R., De Muynck, A., Janssens, P. G., Verdoolaeghe-Van Loo (1972). Correlation of laboratory and clinical data during treatment of leprosy. Ann. Soc. Belge Méd. Trop. 52, 537-548.

Pattyn, S. R., Rollier, R., Saerens, E. J., Rollier, M. R. (1974). Initial three months continuous and intermittent therapy in lepromatous leprosy. A controlled clinical trial. Preliminary data. Ann. Soc. Belge Méd. Trop. 54, 43-49.

Rees, R. J. W., Pearson, J. M. H., Waters, M. F. R. (1970). Experimental and clinical studies on rifampicin in treatment of leprosy. Br. med. J. 1, 89-92.

Shepard, C. C. (1972). Combinations of drugs against M. leprae. Int. J. Lepr. 40, 33-39.

Shepard, C. C., Levy, L., Fasal, P. (1972). Rapid bactericidal effect of rifampicin on M. leprae. Am. J. trop. Med. Hyg. 21, 446-449. 